\section{Ischemic Duodenitis}

Ischemic changes in the duodenum have been reported only very occasionally in patients with severe atheromatous disease of the splanchnic arteries [1-4]. In those cases, the changes were usually chronic and reversed only after revascularization procedures $[3,4]$. We present here a rare case of truly "transient-type" ischemic duodenitis.

A 63-year-old man presented with severe epigastric pain and bloody diarrhea. A few years earlier, when he was 58 , his abdomimal aorta was found to be obstructed below the level of the bifurcation of the renal arteries, due to arteriosclerosis oblit-
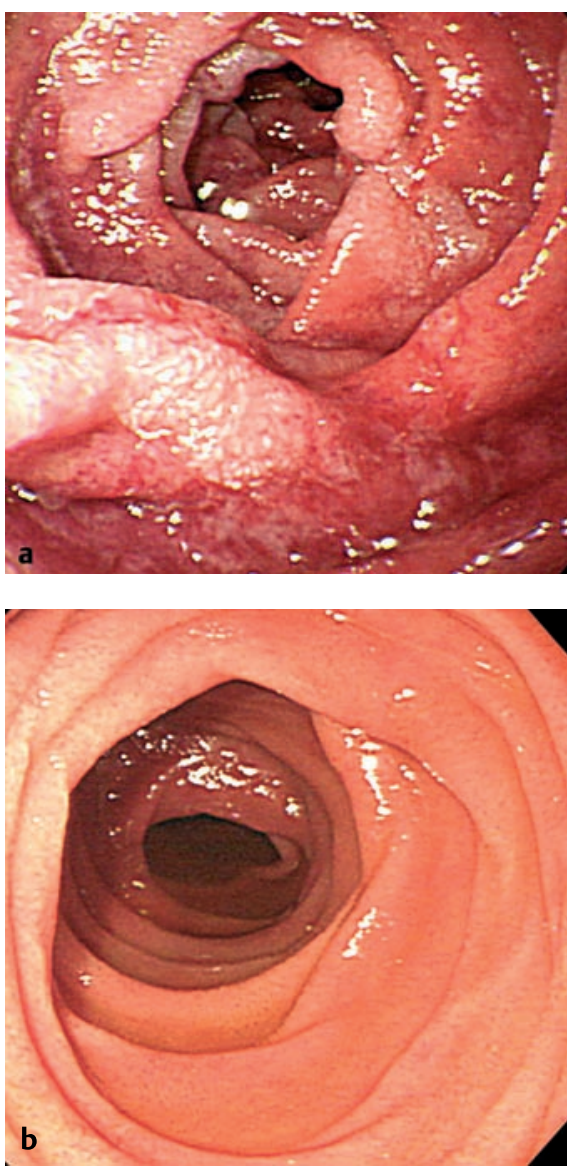

Figure 1 Endoscopic findings in the second part of the duodenum. a On admission to hospital this section of the patient's duodenal mucosa was edematous and hemorrhagic. $\mathbf{b}$ These abnormal findings had disappeared completely by day 7 .

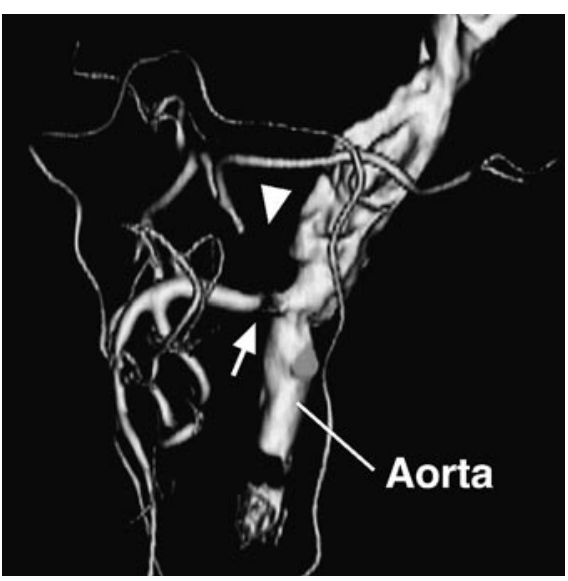

Figure 2 An angiogram using multidetector computed tomography which showed that the celiac trunk was totally obstructed (arrowhead) and the superior mesenteric artery was subtotally obstructed (arrow).

erans. Since then he has been taking anticoagulants. Gastrointestinal endoscopy on admission demonstrated edematous and hemorrhagic mucosa, with multiple erosions, in the second part of the duodenum (Figure 1a). Biopsy showed edematous changes only. Colonoscopy was performed and no abnormalities were found in the terminal ileum, colon, or rectum. Cultures of duodenal fluid and of stool produced no growth of pathogenic organisms and no ova or parasites were observed on stool examination. After a 6day fast, the abnormal endoscopic findings were found to have completely disappeared (Figure $\mathbf{1 b}$ ). Interestingly, an angiogram using multidetector computed tomography demonstrated total obstruction of the celiac trunk and subtotal obstruction of the superior mesenteric artery (Figure $\mathbf{2}$ ), neither of which had been observed at the age of 58 .

Taking these findings together, the transient ischemic changes in the duodenal mucosa appear to have been caused by insufficient arterial blood flow, and these changes recovered spontaneously. The patient underwent a prophylactic stenting operation for superior mesenteric artery stenosis. Since then, the patient has not had endoscopically proven duodenitis for about 1 year, although he sometimes experiences abdominal angina.

\section{H. Seno, S. Mikami, K. Komatsu,} S. Kadota, S. Yazumi, T. Chiba Department of Gastroenterology and Hepatology, Kyoto University Graduate School of Medicine, Kyoto, Japan.

\section{References}

${ }^{1}$ Force T, MacDonald D, Eade OEet al. Ischemic gastritis and duodenitis. Dig Dis Sci 1980; 25: $307-310$

${ }^{2}$ Allende HD, Ona FV. Celiac artery and superior mesenteric artery insufficiency - unusual causes of erosive gastroduodenitis. Gastroenterology 1982; 82: $763-766$

${ }^{3}$ Vaziri ND, Dure-Smith B, Miller Ret al. Pathology of gastrointestinal tract in chronic hemodialysis patients: an autopsy study of 78 cases. Am J Gastroenterol 1985; 80: 608-611

${ }^{4}$ Hojgaard L, Krag E. Chronic ischemic gastritis reversed after revascularization operation. Gastroenterology 1987; 92: $226-228$

\section{Corresponding Author}

\section{H. Seno, M.D.}

Department of Gastroenterology and Hepatology, Kyoto University Graduate School of Medicine 54 Shogoin-Kawara-cho Sakyo-ku

Kyoto 606-8507

Japan

Fax: $\quad+81-75-7514303$

E-mail: seno@kuhp.kyoto-u.ac.jp 